\title{
The Multiperiodicity of $\mu$ Cen (B2 IVe)
}

\author{
D. Baade
}

European Southern Observatory, Karl-Schwarzschild-Str. 2, D-85748 Garching b. München, Germany

Th. Rivinius

Landessternwarte Königstuhl, D-69117 Heidelberg, Germany

S. Štefl

Astronomical Institute, Academy of Sciences, CZ-251 65 Ondřejov, Czech Republic

\begin{abstract}
We re-discuss the reality of the four periods near $0.5 \mathrm{~d}$ and reject the claim (Balona et al., these proceedings) that $\mu$ Cen exhibits a single $\sim 0.66-\mathrm{d}$ period caused by co-rotating clouds. The alleged $0.66-\mathrm{d}$ period is not genuine but results from the simultaneous proximity of this value to the beat period between the true 0.28 - and 0.5 - $\mathrm{d}$ periods as well as to a $2-\mathrm{c} / \mathrm{d}$ alias of the $0.28-\mathrm{d}$ period.
\end{abstract}

\section{Introduction}

The detection of 6 periods in $\mu$ Cen (Rivinius et al. 1998a) and the associated multi-periodically repeating outbursts (Rivinius et al. 1998b) may be important for the understanding of the formation of disks around some Be stars. Meanwhile, Tubbesing et al. (these proceedings) have shown that $28 \mathrm{Cygni}(\mathrm{B} 2.5 \dot{\mathrm{Ve}}$ ) is in its outbursts and multi-mode pulsation probably a close analog so that $\mu$ Cen is not one star of a kind.

Balona et al. (these proceedings) have now put into question that the reported multi-periodicity of the line profile variability of $\mu$ Cen is genuine. Therefore, in the next section, we re-discuss the facts and arguments requiring this multi-periodic solution. Thereafter, we offer an explanation of what circumstances most probably misled Balona et al.

\section{The reality of the multiperiodicity}

Even in observations covering only one week, periods around $0.28 \mathrm{~d}$ and $0.5 \mathrm{~d}$ have over more than a decade always been present. That $\mu$ Cen is at least bi-periodic is, therefore, firmly established beyond any doubt. Note that Aerts (these proceedings) found $f_{\mathrm{A}}=0.56 \mathrm{c} / \mathrm{d}$ and $f_{\mathrm{B}}=0.11 \mathrm{c} / \mathrm{d}$ in the HIPPARCOS observations of $\mu$ Cen. The sampling of these data would not easily permit periods as short as the two real ones to be detected with certainty. But $f_{\mathrm{A}}$ is a 
$1 \mathrm{c} / \mathrm{d}$ alias of the beat period of $0.28 \mathrm{~d}$ and $0.5 \mathrm{~d}$, thereby lending some additional, indirect support to the two basic timescales.

More subtle is the decomposition of the 0.28-d period into two and especially, because of the awkward numerics, of the 0.5-d period into four independent periods (and NRP modes). However, many facts support and even demand such a conclusion:

- Strong $n \mathrm{c} / \mathrm{d}$ peaks (in $\mu$ Cen: $n=2$ ) in a power spectrum may be due to a trend. Mathematically it is, of course, trivial to remove an $n \mathrm{c} / \mathrm{d}$ frequency and its $1 \mathrm{c} / \mathrm{d}$ aliases by subtracting a suitable long-term trend. But the physical justification is lacking: (i) There is no sign of differential atmospheric expansion as, e.g., in hypergiants. Moreover, in a relatively little evolved star, this should lead to large photometric long-term variations, which are not observed. (ii) The HEROS spectrograph is stable to within $0.5 \mathrm{~km} / \mathrm{s}$ (Kaufer 1996), i.e. there is no instrumental drift. (iii) We find no hint of periodic orbital motion.

- The 2-c/d peak and it aliases can be de-composed into 4 sub-peaks. Their separations are roughly $2 x, 1 x$, and $2 x$, respectively, with $x \approx 0.018 \mathrm{c} / \mathrm{d}$. This fine structure prevails also in the $1 \mathrm{c} / \mathrm{d}$ aliases but a frequency of $0.018 \mathrm{c} / \mathrm{d}$ does not appear in the window function.

- Simulations with a single sinusoidal variation at various frequencies near $2 \mathrm{c} / \mathrm{d}$ sampled at the actual times of observation do not lead to a similar frequency splitting, regardless of whether or not a linear trend is added.

- The periods of the first mode yield roughly sinusoidal 'radial velocity' curves. Folding also the profiles with these periods results in phase-dynamical spectra, which in all sufficiently strong stellar lines show the same smooth phase progression across the profiles. That is, the periods can sort more information than has been used for their determination. Moreover, these phase-dynamical spectra are virtually identical for all 4 periods, which is difficult to imagine for false periods.

- The phases are coherent over $\geq 5$ years.

- The two periods near $0.28 \mathrm{~d}$ do not pose any observational or numerical problem. Qualitatively, they share all of the properties enumerated above. Only the quantitative parameters characterizing them are different. In particular, the phase-dynamical spectra imply that the mode indices $\ell, m$ differ between the two groups but are identical within either group.

- Several observing runs from 7 to $\geq 100$ nights with $\sim 100$ spectra each all give consistent results. The shorter data strings could also be reconciled with an 0.33 -d period instead of $0.5 \mathrm{~d}$. However, this ambiguity is perfectly resolved by the long runs.

- The 408 Heros spectra covering more than 3,500 half-day cycles provide a fairly even phase coverage even for a period of $0.500 \mathrm{~d}$.

- Results obtained with different telescopes and spectrographs of different spectral regions all yield consistent results.

- Outbursts repeat every 29 and 54 days, the two main beat periods of the $0.5 \mathrm{~d}$ periods. Over more than 10 years, the times of all line emission outbursts 
observed by ourselves or reported in the literature correspond to within better than a week to the observed and computed times of maximal constructive interference of the modes involved. Since the outbursts are predicted exclusively from stellar lines, whereas they are observed in circumstellar lines, the two data sets are independent. This demonstrates once more the genuiness of the 0.5-d periods and the small differences between them. The completeness of the outburst prediction suggests moreover that, for comparable amplitudes, our de-composition of the 0.5 -d periods is also complete.

\section{Is it cloudy over $\mu$ Cen?}

Balona et al. (these proceedings) analyse a dense series of high-quality spectra. They confirm an $0.5-\mathrm{d}$ variability only with limited significance. This is no surprise: The spectra of Balona et al. were obtained when the combined amplitude of the four 0.5-d variations was, due to their destructive interference, at an absolute minimum (Fig. 1). Balona et al. suggest a period of $\sim 0.66 \mathrm{~d}$ because spectra look alike only every other night.

This, too, is to be expected from the analysis of our own data. $0.66 \mathrm{~d}$ is merely the beat period of the $0.5-\mathrm{d}$ and $0.28-\mathrm{d}$ periods:

$$
1 / 0.28-1 / 0.5 \approx 1 / 0.64 \quad(3.57 \mathrm{c} / \mathrm{d}-2.0 \mathrm{c} / \mathrm{d} \approx 1.5 \mathrm{c} / \mathrm{d})
$$

Note that this same relation also shows that the claimed $0.66-\mathrm{d}$ period is, moreover, a 2-c/d alias of the true $0.28-\mathrm{d}$ period.

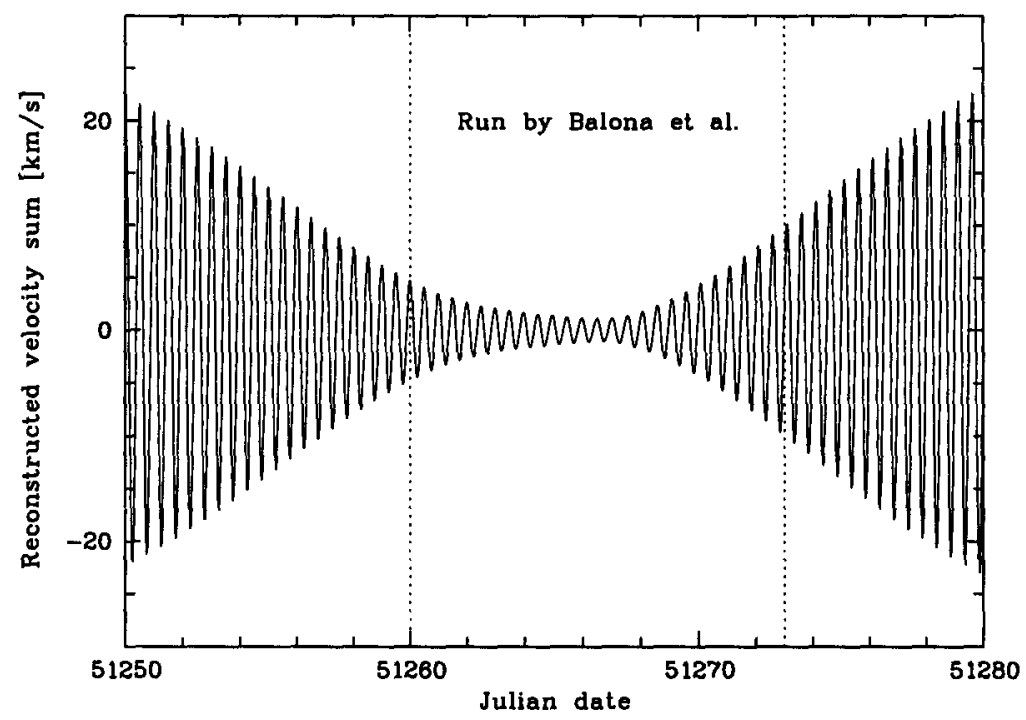

Figure 1. Prediction by the ephemeris of Rivinius et al. (1998b) of the combined amplitude of the four 0.5-d periods at the time of the observations by Balona et al. (these proceedings), which evidently were obtained during a phase of maximum destructive interference 
Balona et al. propose a co-rotating photospheric cloud model with a period of $0.66 \mathrm{~d}$. Balona (these proceedings) discusses analogous models for $28 \mathrm{CMa}$, $\eta$ Cen (see also Balona \& Kaye 1999), and $\zeta$ Tau (see also Balona 1999) and concludes that the concept of co-rotating clouds probably explains the shortterm variability of be stars in general.

However, apart from the probable non-existence of a genuine 0.66 -d period in $\mu$ Cen, any single-period model fails entirely to describe the true nature of this star, which is only revealed in much longer series of observations than the one obtained by Balona et al. The periods of $\mu$ Cen as well as of $28 \mathrm{CMa}$ (cf. Štefl et al., these proceedings) and $\eta$ Cen (Štefl et al. 1995) are phase coherent for at least a decade. Any co-rotating structures would require a very solid 'anchor'. In other stars, this is realized by strong, ordered magnetic fields. However, these properties made the fields (easily) detectable whereas there is zero direct evidence to date (Mathys, these proceedings; Smith, these proceedings) that $\mathrm{Be}$ stars similar to $\eta$ and $\mu$ Cen and $28 \mathrm{CMa}$ do possess magnetic fields.

In summary, all currently available observations do not indicate that, except for the disk and the periodically repeating outbursts, the view towards (of) $\mu$ Cen is blurred by clouds.

\section{Conclusions}

Our multi-period solution for $\mu$ Cen has been shown to be highly robust. A single-periodic co-rototating photospheric-cloud model for $\mu$ Cen is therefore, devoid of any evident observational basis. It is also comforting that at a time, when the reduced $0.5 \mathrm{~d}$ variability afforded a higher sensitivity to other effects, no previously unknown variability was detected. This suggests that, in addition to being quantitatively correct, our period analysis is also reasonably complete.

Not only is the line profile variability of $\mu$ Cen not due to a co-rotating cloud but a detailed study by Rivinius (these proceedings) shows that it can be modeled in considerable detail as multiple $g$-mode nonradial pulsation. Maintz et al. (these proceedings) have demonstrated the same for the apparently single mode of $28 \mathrm{CMa}$. This challenges for this star, too, the cloud model (Balona \& James, these proceedings) with which similar multi-line profile fits have not so far been generated. Any generalized co-rotating cloud model for Be stars at large (e.g., Balona, these proceedings) appears ill-founded.

\section{References}

Balona L.A. 1999, MNRAS 306, 407

Balona L.A., Kaye A.B. 1999, ApJ 521, 407

Kaufer A. 1996, Ph.D. thesis, Landessternwarte Heidelberg

Rivinius Th., Baade D., Štefl S., Stahl O., Wolf B., Kaufer A. 1998a, A\&A 336, 177

Rivinius Th., Štefl S., Baade D., Stahl O., Wolf B., Kaufer A. 1998b, Be Star Newsl. No. 33, p. 15

Štefl S., Baade D., Balona L.A., Harmanec P. 1995, A\&A 294, 135 